\title{
Research on the Teaching of Visual Communication Design Based on Digital Technology
}

\author{
Jianying Bian $\mathbb{C}^{1}$ and Ying Ji $\mathbb{D}^{1,2}$ \\ ${ }^{1}$ Wuxi Vocational College of Science and Technology, No. 8 Xinxi Road, Wuxi, Jiangsu 214000, China \\ ${ }^{2}$ Ajou University, Suwon 16499, Republic of Korea \\ Correspondence should be addressed to Ying Ji; jy@wxsc.edu.cn
}

Received 28 September 2021; Revised 18 October 2021; Accepted 20 October 2021; Published 3 November 2021

Academic Editor: Xin Ning

Copyright (C) 2021 Jianying Bian and Ying Ji. This is an open access article distributed under the Creative Commons Attribution License, which permits unrestricted use, distribution, and reproduction in any medium, provided the original work is properly cited.

\begin{abstract}
In the era of big data, the rapid development of information technology has made the sharing of data and information more free, bringing convenience to the public, but at the same time, the massive amount of data also brings "information anxiety." In this paper, we use the knowledge of design discipline, combined with psychological cognitive science, statistics, and communication discipline, and analyze a large number of excellent cases to effectively combine "information visualization-visual representation-design." The purpose of this study is to enable audiences to efficiently access information through visual symbols in a large amount of data and to increase their interest in reading and satisfaction in accessing information. As a carrier of communication between designers and audiences in the process of visual representation of information visualization, visual symbols can effectively convey information content and emotional concepts to audiences. Finally, based on the theoretical support of the previous paper, combined with our own practical experience, we conduct a systematic study on the application of design thinking and the construction of design methods for the design of visual representations of information visualization. A comparative study on the aesthetics of different categories of APP interface design is conducted, and it is believed that different categories of APP can create distinctive and different categories of APP interface aesthetics through the differentiated design of interface layout, content expression, and visual form, which is considered as the ultimate goal that APP interface design must pursue. Visual representation is a method and means to realize information visualization as a representation practice that expresses the meaning of information in the form of visual symbols. The visual representation of information visualization uses visual symbols as a medium, and the audience interprets the visual symbols based on cognitive experience to obtain information, which helps to maximize the dissemination of information.
\end{abstract}

\section{Introduction}

In the context of the "big data era," big data, supported by mobile Internet and information technology, has been widely used in many fields and has become one of the core resources affecting social development. The sharing of data and information is becoming increasingly flexible and diverse, and information is becoming globalized [1]. Faced with the language communication barriers between different ethnic groups and cultures, a communication method that allows information to flow freely is needed to facilitate communication between various groups and cultural exchanges between different countries [2]. In recent years, the number of people and web pages on the Internet has been on the rise, and the amount of data and information has been increasing, but there is a lot of information but it is complicated and disorganized, people have to passively receive thousands of pieces of information every day, and too much data will easily cause "information anxiety." Under the double pressure of information explosion and fast-paced life, people tend to read in fragments, and the mode of reading is changed from text to map, and the purpose of reading information is also clearer [3]. However, nowadays, the means of information processing obviously cannot keep up with the speed of information growth, and the traditional way of information dissemination can no longer meet the needs of the public and the 
continuous development of society, so how to quickly obtain valuable information from the massive amount of information has become the focus of most people's attention [4]. The main problem facing information disseminators is how to quickly catch audience's attention, effectively disseminate information, reduce the visual pressure of the audience, eliminate visual barriers, and make the information receiver quickly and actively obtain information. The current design forms for information dissemination focus too much on the design symbols, ignoring the psychological needs of the audience and the information dissemination process, resulting in the failure to maximize the dissemination of information [5].

Information visualization visual representation communication design uses visual symbols as a mediator, giving the designer the dual identity of information designer and visual designer to communicate information, which is more in line with today's needs [6]. Visual communication design is a process in which a designer conveys a specific message to the communicated object through a visible form of artistic expression in order to achieve the desired purpose, thus having a certain influence on the communicated object. Visual communication design is composed of three main elements: text, illustration, logo and other visual symbols, communication, and design. Visual communication design is divided into several parts according to design science, such as type design, display design, packaging design, and logo design [7]. Visual communication design has been developed from the western graphic design (graphic design), and the development of technology has brought people new visual experience, and visual communication design has emerged accordingly. Visual communication design started relatively late in China, but with the rapid development of China's economy in recent years, the huge demand of the market has stimulated the rapid development of visual communication design teaching [8]. The goal of visual communication design teaching is more accurate, the design of teaching courses and contents is more perfect, and the investment in teaching facilities is constantly strengthened, in order to cultivate more qualified visual communication design talents for the society. The teaching of visual communication design in China, from a new starting point, can better meet the market requirements and develop in a clearer direction by combining with new science and technology [9]. Therefore, at present, China's visual communication design teaching must seize the opportunity to promote its own rapid and healthy development with new design concepts and the application of new technologies combined with the needs of social development. As an important content of design teaching, visual communication design has been developing rapidly in China in recent years, and it is constantly updated in terms of teaching philosophy, teaching methods and teaching equipment, actively combining with other majors and insisting on the long-term development path of diversification and modernization [10]. Digital technology, as a new way of artistic expression, has prompted visual communication design to enter a new stage of development. The maturity of digital technology makes the digital expression of visual communication design a new trend in the field of visual communication design. The digitalization of visual communication design has prompted designers to design through multidisciplinary cross-thinking and multiple design methods, and design strategies and thinking are constantly evolving [11]. Through digital technology and media, it can not only enrich designers' expressions and provide diversity of artistic expressions but also enhance the participation and interaction of information receivers and improve the effectiveness of information communication, as well as promote flexible and broader choices of communication methods.

In the context of an increasingly digital living environment, digitalization has become an important part of popular culture, and the interactive development of visual communication design from static to dynamic makes it a digital art with its own uniqueness. The digital expression of traditional visual design reflects the infinite charm of combining art and technology and represents the new development direction and trend of future design art. In this paper, we clarify the concepts of information visualization and visual representation and discuss the communication mechanism between information visualization, visual representation, and visual communication based on Michel's "quadrilateral representation system theory" and Stuart Hall's "encoding and decoding" theory. Secondly, visual symbols, as a carrier of communication between designers and audiences in the process of visual representation of information visualization, can effectively convey information content and emotional concepts to audiences. In addition, the application principles, application forms and shapes, and types of application of visual representation of information visualization are studied with typical cases; finally, based on the theoretical support of the previous paper, combined with its own practical experience, the design of visual representation of information visualization is systematically studied in terms of design thinking application and design method construction. On the basis of the research results of this paper, based on the analysis of the online questionnaire report, the visual representation design of information visualization is carried out to disseminate health protection information based on "health protection knowledge," which is of public concern, to verify the feasibility of the previous research.

\section{Related Work}

Any form of artistic expression is created on the basis of social and technological development, and technology can promote the development of art [12]. Digitalization is the basis of computer, multimedia, Internet, and information society technology. The arrival of the digital era has brought new forms of artistic expression to social life, and the widespread use of digital media has also changed the way people receive and disseminate information. The development of digital technology has also brought changes to visual communication design in terms of design methods and processes, and visual communication design based on digital expression has characteristics such as rich information content, comprehensiveness, and interactivity [13]. Visual communication design in the digital era adopts various forms of information expression such as sound, graphics, and text 
and adopts a lot of computer and multimedia technology, such as Photoshop and other graphic processing software, and the design works can realize the interactive communication between the sender and the receiver [14]. At the same time, the medium of communication has also expanded from traditional books, newspapers, and TV to mobile phone MMS, mobile phone newspapers, and digital media such as network advertising and electronic display. The design software can be used to create interactive communication between senders and receivers.

The digital visual communication design process also has new characteristics, from the basic design definition by the designer according to the design purpose and the basic information to be expressed, to the determination of the information content to establish the overall information architecture, to the selection of suitable expressions according to the information using digital technology for digital processing design, and finally, to the combination of the corresponding software and hardware equipment to communicate the design results through digital media. Digitalization puts forward new goals for the teaching of visual communication design, requiring the cultivation of visual communication design talents who use digital expression as the main means of expression as the fundamental goal, and the cultivation of students' innovative consciousness and digital concepts [15]. At the same time, digitalization also puts forward new requirements on the concept, content, and method of teaching visual communication design, and digitalization inadvertently introduces the "diversity" of expression and "individualization" of communication content into the teaching of visual communication design.

First of all, digital expression makes the scope of teaching visual communication design more extensive, so that it extends from traditional graphic design, industrial design, product design, etc. to the scope of computer networks, with stronger professionalism. The teaching of visual communication design should not only focus on the traditional theoretical teaching and professional basic education but also on the application and operation of digital technology. Secondly, from the point of view of teaching methods, the traditional teacher-led lecture method has been difficult to adapt to the requirements of the digital era, based on digital visual communication design teaching must be combined with teaching and practical operation, and focus on the cultivation of students' hands-on ability on the basis of theory, so it should take more emotional experience, case teaching, and problem teaching methods, through the interaction between teachers and students and students and society, and make full use of multimedia, the Internet, and other digital technology [16]. Therefore, we should adopt more methods such as emotional experience, case teaching, and problem teaching, through the interaction between teachers and students and students and society, make full use of multimedia, Internet, and other digital technologies for teaching services, and improve the effect of visual communication design teaching through effective methods and advanced auxiliary equipment [17]. At the same time, we should also pay attention to the graduation design of students should be closely related to the needs of the times and try to improve students' ability to use various kinds of knowledge comprehensively, so as to achieve the effect of comprehensive development of theory and practical ability.

In order to adapt to the digitalization of visual communication design, we should pay attention to cultivating students' understanding and mastery of visual language, their unique understanding of design language from an artistic point of view, and their ability to use it flexibly and skillfully, as well as cultivating students' practical operation and visual expression ability, so that they can master digital technology and use digital hardware and software for artistic design. Visual communication design, as a branch of art and design, must be based on art [18]. Therefore, the teaching of visual communication design must pay attention to the teaching of professional foundation courses, actively study the curriculum design and teaching methods of foreign visual design teaching, integrate and reconstruct the basic design courses, strengthen theoretical teaching, and lay a solid professional foundation for art design [19]. Although the digitalization of visual communication design is very technical, the basic courses are the basic guarantee for design, and we still need to pay attention to the teaching of professional basic courses such as sketching, design color, and type design. At the same time, the digitalization of visual communication design is a modern art design supported by technology, with strong practicality, and its use must be closely integrated with modern digital technology and digital media [20]. Therefore, the teaching of visual communication design should pay attention to the cultivation of students' technical operation ability and improve their comprehensive design ability while focusing on the teaching of art foundation [21]. Especially in the current context of numerous digital media and complex hardware and software devices, the teaching of visual communication design must strengthen students' ability to use computer, Internet, and multimedia technologies for artistic design and master the methods and skills of using common design software and hardware devices.

\section{Digital Design Visualization}

3.1. Visual Visualization Theory. Cognition, the process by which people acquire knowledge, apply knowledge, or process information, is the most basic human mental process. It includes sensation, perception, memory, thinking, imagination, and language. According to Bruner, a pioneer of cognitive psychology, cognitive representation is the process by which an individual perceptually converts objects or events in his or her external environment into internal mental events. In this paper, the theories of visual cognitive representation in information visualization have been studied, including "inner picture theory," "natural association theory," "custom theory," "memory principle," "memory theory," "memory principle," and "emotional design principle." Visual flow refers to the natural visual flow habit of people when they read, usually from top to bottom, left to right, and clockwise from top left to bottom right. Indicative visual symbols will guide the viewer to read according to the visual order and enhance the fluency and interest of reading. The audience can understand the process of soft drink manufacturing more 
clearly and intuitively. Visual misperception means that when an observer observes an object, based on empiricism or under the interference of objective factors, he or she makes a wrong judgment and perception of the object that is not in line with the objective facts. The German psychologist Hermann Ebbinghaus used circles of the same size placed around two groups of circles of different sizes to produce the misperception effect that the left and right sizes of the central circle were different (as in Figure 1), revealing the phenomenon that the brain incorrectly parses visual information.

The purpose of information visualization is not only to make the audience understand the information but also to make the audience remember the information as much as possible, such as the visual view of science and map, which can help the audience to remember effectively in daily life. In the visual representation of information visualization, the use of the "four rules of things" and "combined memory" can help audiences remember information better. According to George Mandler, the human brain can remember information in categories, and if there are less than three pieces of information in a category, people can easily recall them. The more information there is, the lower the efficiency of memory. For example, in our life, we often divide communication numbers into several groups to remember, a year into four seasons, each season into three months, so in the visual representation design of information visualization, try to divide the information into four categories, within four groups, to facilitate the audience to remember. When the audience gets information, it will be easier to remember the new information that has a connection with the existing information. In addition, a certain repeated information will stimulate the neurons in the brain, and the audience will therefore form a long-term memory. For example, repeating a keyword in a view not only emphasizes the topic but also stimulates the audience and creates a longer-term memory. In the process of visual representation of information visualization, i.e., the practice of visual representation, not only the content is represented but also the emotional design concept is included. The designer conveys the design concept and emotion to the audience together with the visual symbols, and the audience understands the message and the meaning behind the visual symbols through the visual symbols with their own experience and self-perception.

\subsection{Analysis of Information Visualization Visual Representation} Applications. Being able to accurately convey information is the basic principle and characteristic of the design application of visual representation of information visualization, and it is also the primary premise for visual representation of information visualization to realize information dissemination. Content refers to all the graphics, text, and other symbols related to information presented in the information view. Accuracy does not refer to the level of accuracy required of the information, but to the fact that the information conveyed is errorfree, trustworthy, and inferred, and the content has a basis, source, or scientific nature. Content accuracy is based on the subject matter of the information, the purpose of information dissemination, and the needs of the audience and is the main thread running through the design of the visual representation of information visualization and the key to the information conveyed by the information view. The reason why an excellent information visualization view can attract audience's attention, draw audience's attention, and thus enhance the efficiency of information delivery is that it has a strong visual aesthetic and expressive interest, which can catch audience's eyes and regulate their emotions under the same thematic information expression. As shown in Figure 2, the right picture is presented with more vivid visual symbols, which can better attract audience's eyes, eliminate the fear emotion, read and identify better, and can better promote the protection knowledge. The image uses curved shapes, arrows, and other visual symbols to represent the process from planting-picking-transportation-processing-sales-consumption-packaging and recycling, so that the audience can more clearly see.

The functionality of information visualization is the sum of audience's perceptual, nonutilitarian aesthetic judgment, and the overall psychological impressions and functional feelings gained in the process of use based on the reliability and usefulness of the visual representation symbols. Information visualization is a way to sort out complex and esoteric data information into more organized and easily readable information through visual symbolic representation. The world coffee and tea consumption per capita chart introduces the per capita consumption of coffee and tea in 20 major countries, refining coffee and tea into graphic symbols and adopting a dispersive arrangement for visual representation, which is aesthetically pleasing in form and allows the viewer to access the information more intuitively and efficiently. Dynamic design is an inevitable trend in the future development of visual communication design. Through new digital media technology and communication media, the design content is richer, the message is more communicative and innovative, and the visual communication design becomes a new visual form. Dynamic design also means the mainstream trend of visual communication technology, that is, according to the current mainstream aesthetic to make changes in the technical level and designers in the design of products to ensure that the maximum degree with the times, in any period of time, product design will not be separated from the subjective aesthetic of the masses, which is one of the reasons for the vitality of visual communication technology. Designers should fully understand the aesthetic tendencies and laws of user's senses before designing a product and fully consider whether it will cause a sense of impact due to visual differences or a sense of indifference due to aesthetic fatigue, and only under the premise of good prep work can product information be accurately conveyed to the masses. Nowadays, people have a strong sense of boredom and discomfort for the flat and cluttered information images, so the important information screened out is placed at the top of people's sight, and images and animations rich in dynamism and strong colors are highlighted from the surrounding information to mobilize people's curiosity and attention for exploration (as shown in Figure 3). It breaks into public's field of vision with bold and active gestures, provides more possibilities for visual choices, enhances the degree of stimulation of public's visual reasoning and physiological level, and creates the 


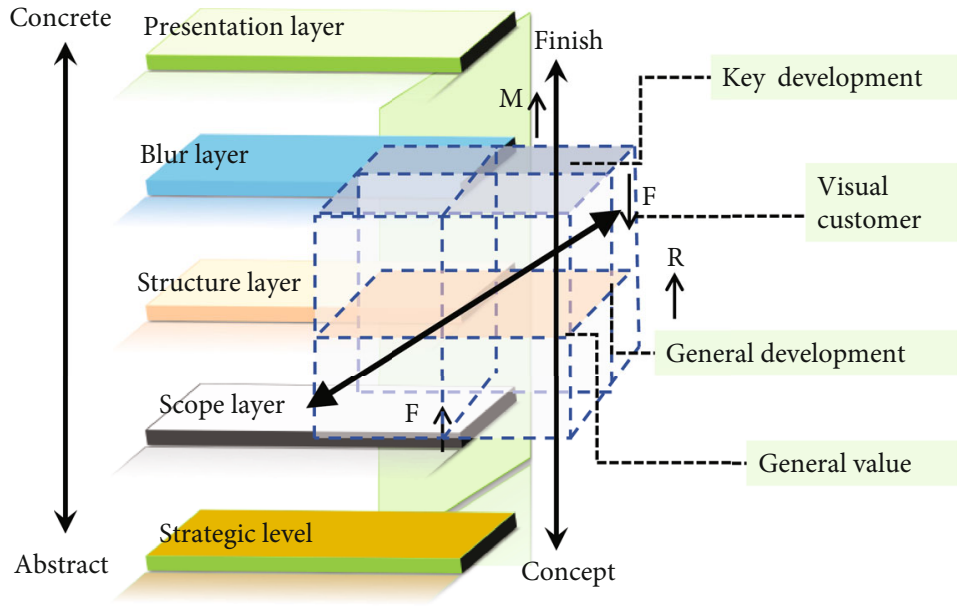

Figure 1: Visual illusions.

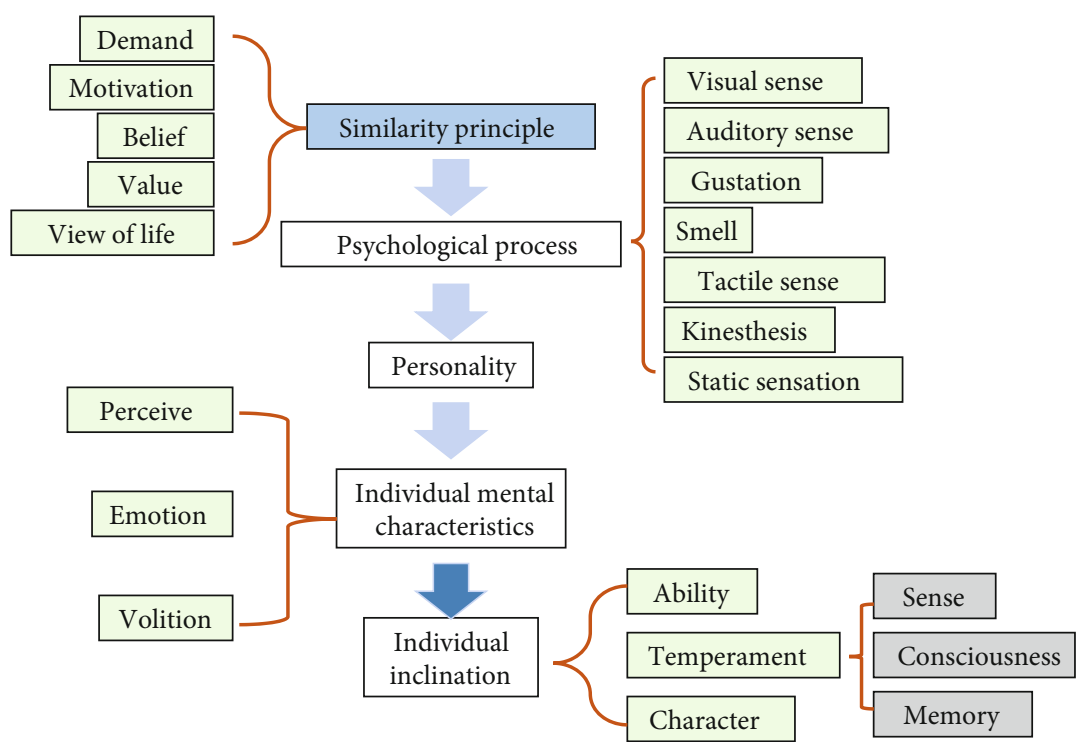

FIGURE 2: Similarity principle relationship diagram.

priority prerequisites and necessary conditions for the rapid spread of images in the tide of information. The dynamic design of visual communication establishes an effective connection with public's mind at the early stage of communication and communicates with graphics. The design of some successful logos emphasizes the intrinsic characteristics of the form, concentrates the essence of the meaning, strongly activates the key clues closely connected with the theme, filters the irrelevant and superfluous graphics automatically, and enhances the efficiency and permanence of the visual communication information, and dynamicization becomes the future. The development trend is dynamic.

In the digital era, visual communication design bids farewell to the traditional flat paper printing, to bring a new experience to customers visually, and to start from meeting their visual needs, which is the embodiment of the humanistic concept of visual communication design. Designers create works with a strong human touch through digital technology, also in the hope of seeking people's spiritual solace in the contemporary fast-paced, high-stress social environment, relieving fatigue, and releasing pressure has become the key to design inspiration, touching the sensitive nerves of people's souls, opening the mouth of emotional catharsis and expressing emotional breakthroughs, providing effective services for people's psychological demands in a comprehensive manner, reflecting as much as possible humanistic care, to meet the emotional needs of the public. For example, some public service announcements reflecting "love," from the heartwarming advertisement in which a child brings footwash to his mother and cares for his parents to the warm advertisement showing brother and sister's love and mutual help abroad, all of which reflect a strong sense of human feelings, so that every viewer can take the initiative to care for their closest family members after watching them, etc. This way of sending human feelings is the embodiment of the application of visual communication technology at the 


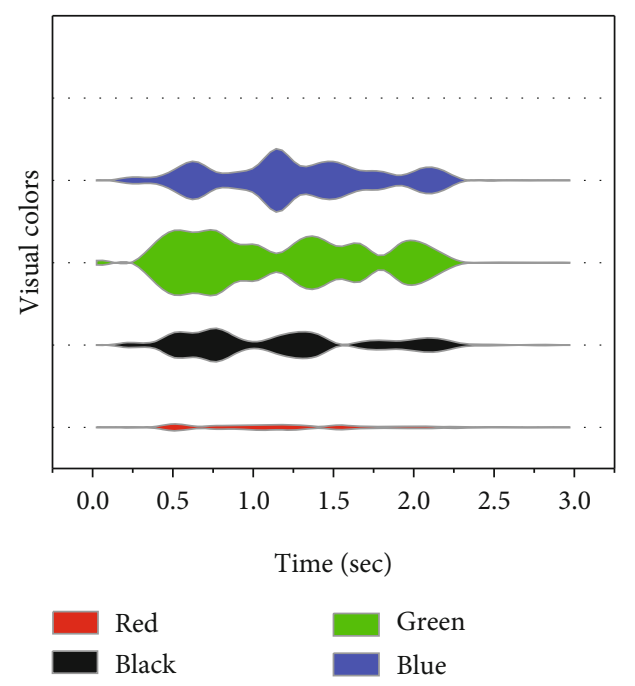

Figure 3: Dynamic display of visual colors.

cultural level, and it is also the need of modern society to evoke a sense of belonging to each user's family. Each country, each nationality in the culture although there are more obvious differences, but family, friendship, love, etc. are human forever unchanging topic; the expression of emotional appeal and trust is a visual design can be preferred a major theme, both can resonate with the user in the emotion, and even designers in the creation will also create a better inspiration in the selection of topics and the use of digital embodiment of warmth, to achieve the Win-win purpose, so in today's cold data-based era, warm emotional expression seems warm and heartwarming, which is also a lever to balance technology and emotion.

3.3. Diversity of Visual Communication. Nowadays, electronic technology and digital media are developing rapidly, and the design field is expanding. The design characteristics of visual communication design are developing in a diversified trend, which mainly also includes different countries and different national cultural needs. The design of cultural elements of various countries and nationalities is included in the image, which promotes the cultural exchange and interaction between countries and leads a young new era of visual aesthetic field (as shown in Figure 4). In the field of civilian design, visual language presents people with different expressions, graphics are flat, images are dynamic three-dimensional, and what they pursue is a broad international vision to make the design with superb quality, thus expressing the inner language logic. The creation of graphics or images, the thinking space of thinking, and the degree of public acceptance are all based on the rational cognition of globalization and diversity, and different countries and nationalities should be consistent in the design of graphic language in modern design expressions. The law of change is extremely important. At the same time, designers should also maintain a keen thinking and advanced aesthetic vision of visual design, look at the transformation of graphics from a professional point of view, look for interest in the creative process, constantly go beyond the established design goals of

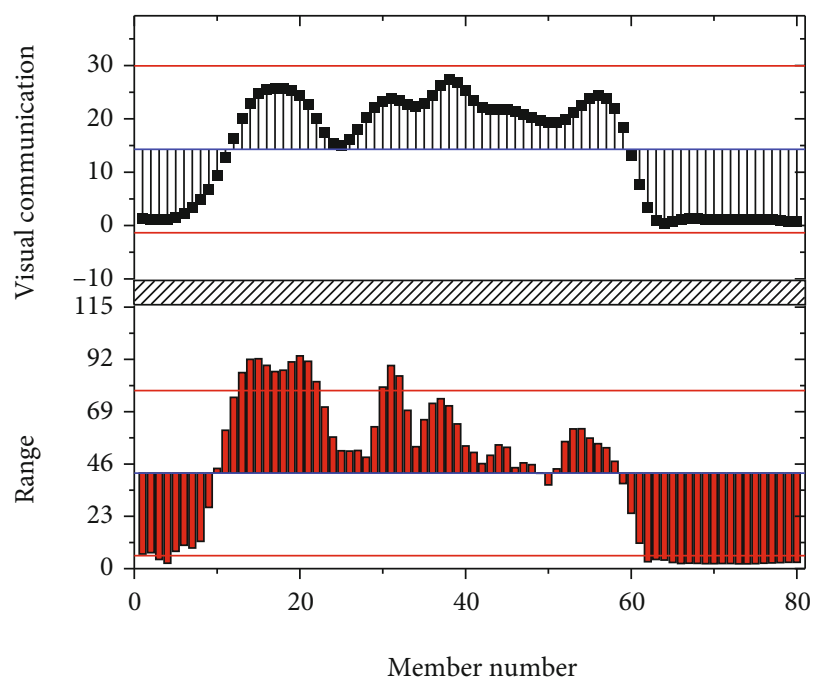

FIgURE 4: Changes in visual communication.

the past, and always maintain a unified pace with modern visual design, and it is especially important not to deviate from the track of modern design.

Visual communication design itself is a discipline that includes a variety of fields; modern visual communication discipline is no longer simply graphic design graphics; it is through the visual design performance and other media to convey to the audience; the distinctive characteristics of the times and the connotation of the times are reflected in its performance. With the continuous progress of modern technology and the use of new energy sources and the expanding market for the development and application of product materials, etc., a new field of design has been formed in which visual communication is interrelated with other visual media in collaboration with each other. In today's highly civilized and developed design, visual communication design is also constantly developing and progressing, and public's requirements for visual communication are no longer just at the simple physical level, but include psychology, science, and other disciplines mixed together in the dynamic graphics or images; visual communication today is also constantly exploring new values in different fields, so designers are required to pursue new thinking space. The design of visual communication is not only limited to the structure of two-dimensional space, such as three-dimensional space or four-dimensional space.

\section{Ease of Use of Digital Visual Communication}

Digital color in the interface design, through the human visual sense to receive information, conveyed to the human brain and then affects the human psychological activities; for the user, color is an important factor affecting the use of the interface to give psychological stimulation. For different APP theme positioning, the application of color must be in line with the theme, or calm, or sunshine, appropriate interface color matching can more strongly attract user's eyes. A cell phone APP facing different user groups but also according to the gender range of the crowd, age range for 
different color applications, and color like human to convey the message has never been very intuitive and warm. The stimulation and influence of color to the user happen very quickly; the form of color information transmission such as color matching, hue primary and secondary as well as hue brightness, color matching, and saturation will have an impact on user's senses and psychology; each color has its own unique properties; different combinations of color properties applied to the interface design will also produce different sparks, bringing different psychological impact to the user. Each color has its own unique properties, and different combinations of color properties applied to interface design will produce different sparks and bring different psychological effects to users (as shown in Figure 5). In APP interface design, when the color is not properly applied, users may have visual fatigue or psychological discomfort, and this negative psychological activity may prompt users to stop browsing the interface or give up the use of APP; after all, most of the human choices come from the psychological reflection. Different colors and color matching will bring different psychological reactions to users, and different color attributes symbolize and express different messages, such as yellow for sunshine, green for hope, and red for enthusiasm. Designers should also use the attributes of colors and emotional factors to match the application of user's psychology, so as to better meet user's psychological needs and emotional needs. A smart designer must use the properties of color to make the APP interface suitable for the user, and in line with user's psychology, the first thing to consider is user's visual aesthetic feeling; as color has visual comfort, the application of the color should start from user's visual experience, to bring the user a comfortable and harmonious visual feeling. With the development of modern society, people are finally moving to a whole new world; digital development is no longer an unfamiliar field; visual communication design has also entered a broader and broader category; print design, written design, video design, book design, display design, visual environment design, etc., are indicating a new design concept is coming.

Under the background of digital era, technology is developing rapidly, people are in the state of data explosion electronic market competition fierce society; various functions of cell phone APP is widely used by people; mobile clients to user's information delivery methods are also growing in a diversified trend. The interface of mobile APP has also become one of the conditions of user's demand for use. In the face of today's developed technology and the competitive environment of APP market, user's demand for product use, emotional demand, and psychological demand are gradually increasing; the way of APP world to deliver image information to users can no longer continue to be limited to the static two-dimensional graphics in traditional communication methods; the way of application of graphics in interface design should be changed from traditional two-dimensional static graphics to rich and dynamic kinetic graphics with more information, which can bring users a bright new way of communication in terms of visual presentation effect. Compared with the monotonous traditional flat graphics, multidimensional kinetic graphics bring users rich and lively visual induction while adding interest to user's use process and enhancing user's desire to use, and the novel kinetic graphics applied to the interface design of APP can also play a good role in promoting APP. In the sea of visual communication, motion graphics, as an important form of expression, has officially entered the application of interface design; due to the high-speed operation of modern society, cell phone APP has just become one with people and become an inseparable part of people in the busy, mechanical work and life, when the interface of APP incorporates the performance of motion graphics to cause a new wave of using APP. After the market receives the strong positive feedback from users, more energy and support will be put into the interface design of APP (as shown in Figure 6). The dynamic presentation of graphics is the most representative and feedback expression in the process of visual communication of human-computer interaction, and kinetic graphics are different from animations in that kinetic graphics change dynamically through user's operation instructions, and user's visual feedback comes from his own operation, with the user as the subject of his own instructions.

Dynamic graphics convey information in a dynamic way, so that users can receive and perceive the visual language conveyed by the APP interface more quickly and comprehensively, and it can analyze the process of user interaction with the APP interface more intuitively. Usually, the cyclic visual elements are applied in the interface as the expression form to deepen user's impression of use, increase the interest of the interaction process, and attract user's attention. The mobile APP and the user were not in the same dimension, but due to the emergence of kinetic graphics, it strengthens the interaction emotion between the user and the mobile APP, bringing the user a more intuitive and operational interaction feeling improving the quality and efficiency of the information transmitted by the interface. In APP interface design, graphic elements that can bring users good visual sense are the basic premise of making motion graphics, and suitable motion graphics can make users enhance the overall communication sense with APP (as shown in Figure 7). The loading graphics are processed by kinetic effect, which can realize the loading function without bringing the user a sense of fluent stuttering. The information conveyed to users by motion graphics is threedimensional and multidimensional, and it plays a good role in guiding users' interface operation while arousing their interest in using it, and it brings the interface closer to users in the process of guiding them, which is the most real feedback of human-computer interaction.

For mobile APP interface, motion graphics are multidimensional for the information conveyed by the interface, and motion graphics are a very rich and effective way to convey visual information. In the APP market, the application of kinetic graphics interface design directly distinguishes it from other APPs that are stagnant in traditional graphic applications. The ease of use of motion graphics enables users to receive information more intuitively during their use. User's pleasure is enhanced by the diverse and dynamic changes. The significance of the application of motion graphics is reflected in the four aspects of enhancing the 


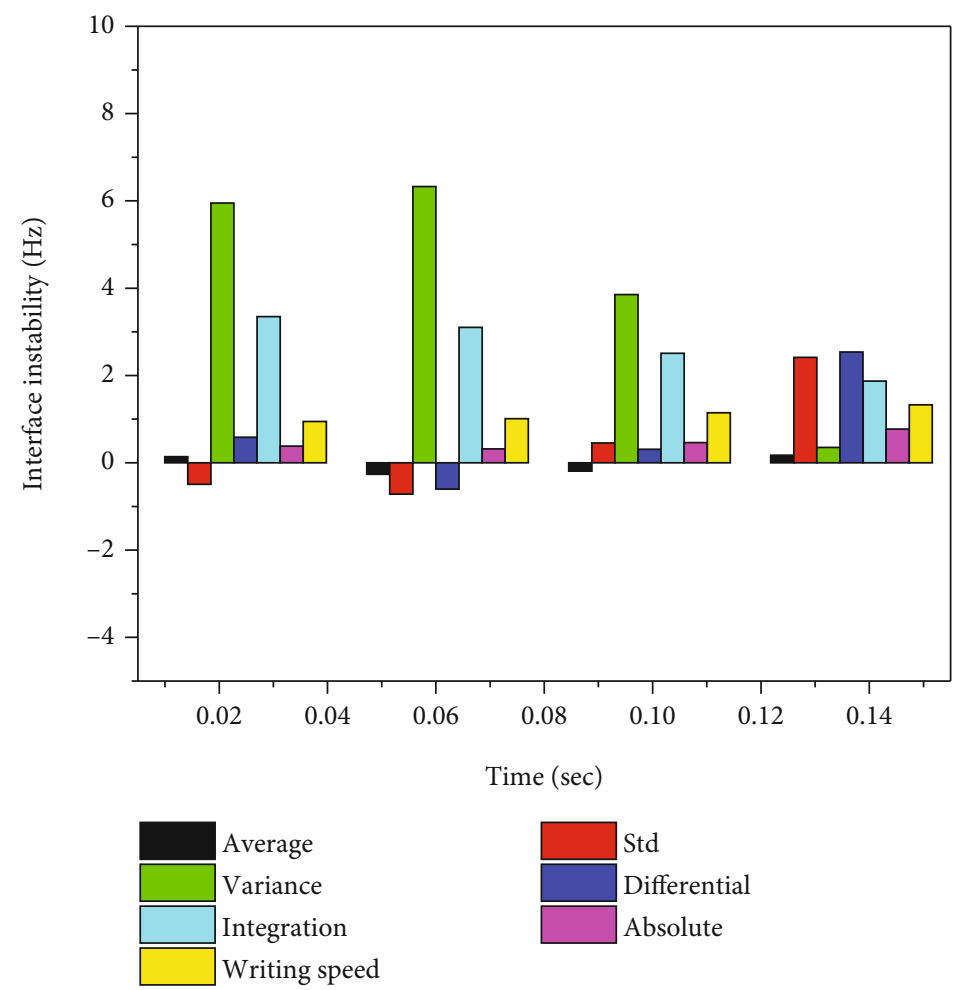

Figure 5: Impact of different interface factors on users.
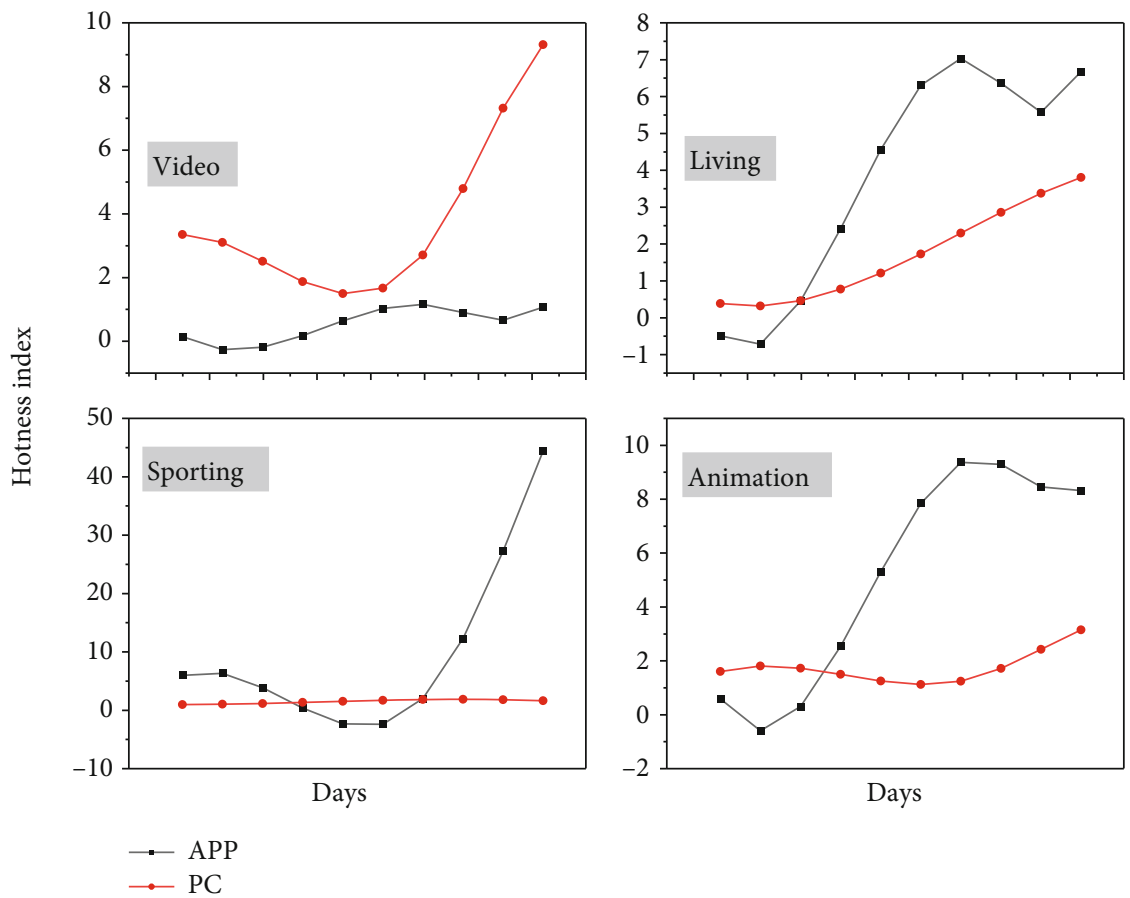

Figure 6: The change of the hotness index of visual design.

contrast, intuitiveness, uniformity of change, and pleasure of the interface function. Reasonable application of kinetic graphics can show the system of APP clearly to the users, divide and show the functions of major modules in APP interface and the hierarchy of modules clearly, make the function switching and module transition between them more natural, and enhance the aesthetic height of the whole APP interface. The expression form of kinetic graphics is 


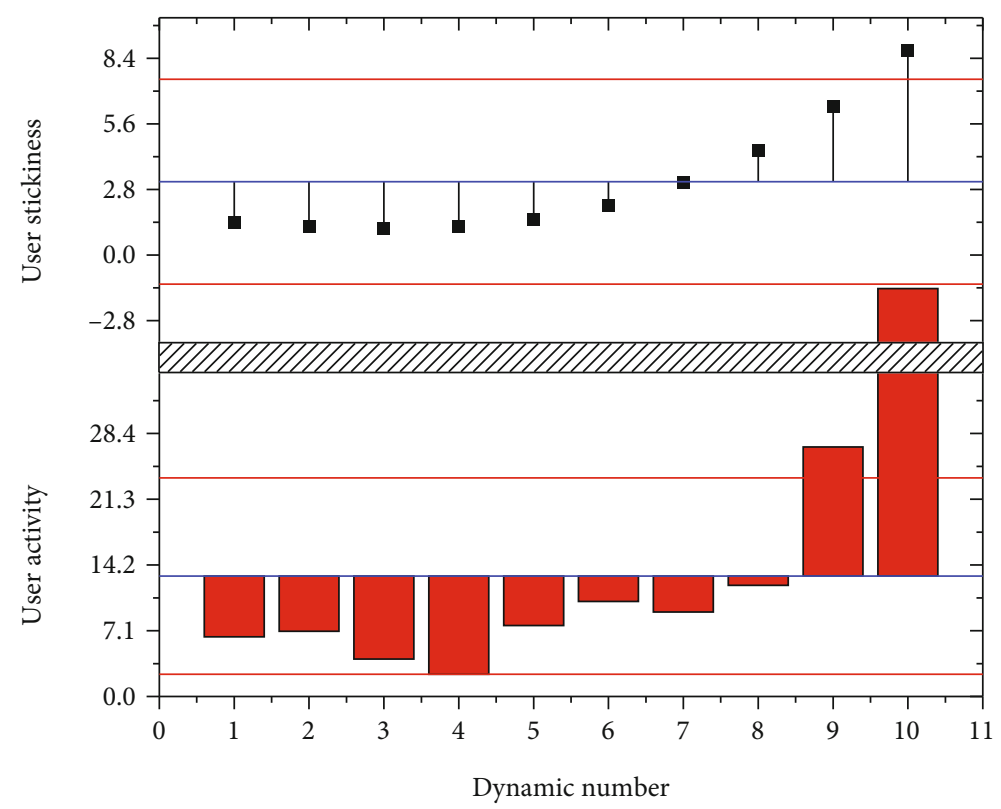

FIGURE 7: Comparison of user stickiness between dynamic and static graphics.

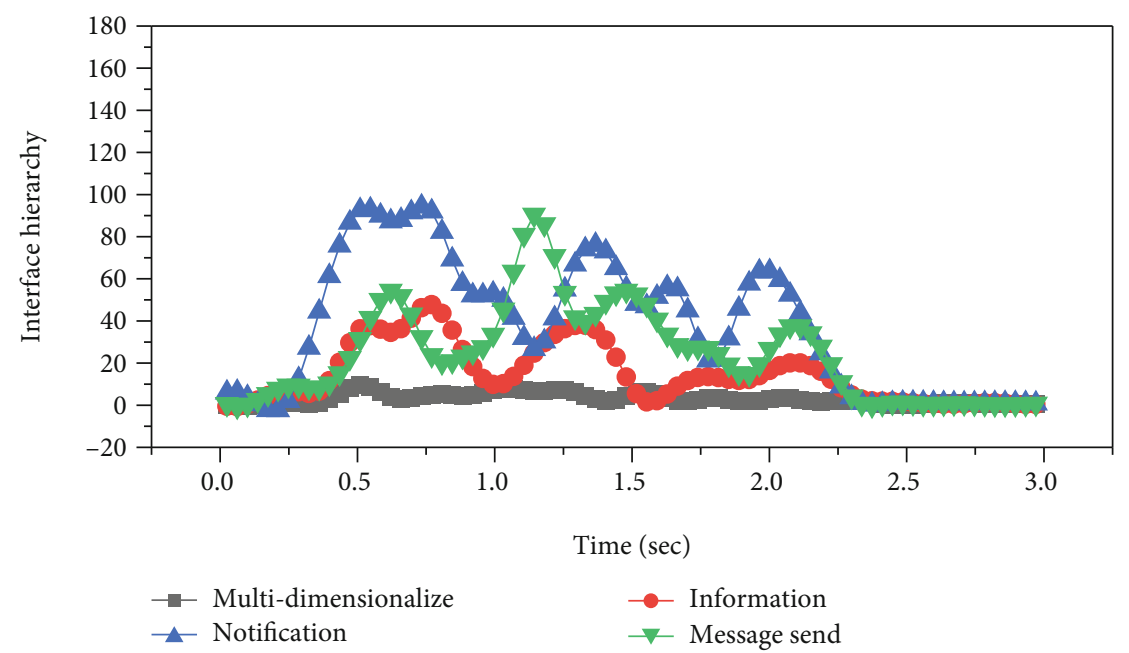

Figure 8: Relationship between interface hierarchy and functionality.

flexible and versatile, which can contrast the theme and design concept of APP interface design with other same functions in a unique and novel way to form a huge difference, compared with APP using traditional interface, the application of new graphic form is more able to enrich the overall content level of interface design (as shown in Figure 8). The emergence of kinetic graphics adds a time dimension to the interaction between users and the APP interface, so that users can feel the precise control of the kinetic graphics in the process of feeling and experiencing the operation of the APP interface, so that users can better understand and remember the functional system of the APP interface. The kinetic effect graphics can multidimensionalize the information conveyed by the APP interface, and the dynamic expression can clearly elaborate the multi- level relationship in the interface and the way of interaction between the interface and the user, which can intuitively transfer the information to the user, enhance user's real sense of use, and alleviate user's doubts.

In the background of digitalization era, various electronic mobile clients have occupied the majority of people's life and work. For mobile APP, under the basis of achieving thematic positioning and functional utility, how to make itself more special and how to attract users' attention are also important factors. So in today's situation, the interface design of APP needs to inject more emotional factors, which can take users' emotions as the starting point of design and focus on the emotional integration of interface design. The addition of motion graphics can not only make users experience rich emotion and pleasure during the operation of the 
interface but also make users feel the good intention of APP interface designers. The fun and flexibility of motion graphics will certainly leave a deep impression on users during their first use, and the pleasure and emotional resonance given to users in the process of use is incomparable to traditional two-dimensional graphics. In the operation process of the APP interface, the dynamic effect graphics establish a good information transfer link between the user and the interface through the dynamic and novel form, and the user can enjoy the fun of the operation and receive the information from the interface quickly. The user can establish an inseparable relationship with the interface in a relaxed state, which enhances the reality of the situation while the user interacts with the interface, generating positive reactions and making the user feel happy while achieving functional satisfaction. The diverse and three-dimensional expression of flexible and interesting motion graphics is a seemingly intangible and tangible pleasure experience for users. The dynamic forms presented by different motion graphics are different, because the movement process of the object is very complex and changeable, so its movement pattern is also varied, through the visual presentation of motion graphics, so that the user can receive the information transmitted by the APP interface more clearly and understand the content of the APP interface more intuitively. Different forms of dynamic graphics can express different interface information logic and information hierarchy to users, so in-depth analysis of the presentation of dynamic graphics can help to better reflect the functionality of dynamic graphics.

\section{Conclusion}

By analyzing the background conditions of the digital era, this paper takes cell phone APP interface design as the research object, focuses on the current situation of APP interface design under the current situation, and analyzes the aesthetic characteristics of visual elements of APP interface design. This paper believes that among the visual elements of APP interface design, graphic elements can express the imagery of APP interface, text elements can intuitively state the information content of the interface, and color elements can help the interface to convey emotions to users. In addition, the application principles, application forms and shapes, and types of application of visual representation of information visualization are studied with typical cases; finally, based on the theoretical support of the previous paper, combined with its own practical experience, the design of visual representation of information visualization is systematically studied in terms of design thinking application and design method construction. The criterion to determine whether an APP is successful is the degree of stickiness brought to users, and the first sensory experience of users to APP can determine the degree of user viscosity. A comparative study on the aesthetics of different categories of APP interface design is conducted, and it is believed that different categories of APP can create distinctive and different categories of APP interface aesthetics through the differentiated design of interface layout, content expression, and visual form, which is considered as the ultimate goal that APP interface design must pursue.

In the future, to make the text of APP interface can both convey the inner meaning to users and bring novel visual impact to them, the differences are felt by comparing three-dimensional text with flat text and regular font with special font, and different aesthetic interests of different user groups are created according to different charm of text.

\section{Data Availability}

The data used to support the findings of this study are available from the corresponding author upon request.

\section{Conflicts of Interest}

The authors declare that they have no known competing financial interests or personal relationships that could have appeared to influence the work.

\section{Acknowledgments}

The study was supported by the "Research on the Application of Aesthetic Thinking in the Design and Creation of PowerPoint Courseware -On the Path of Aesthetic Education in Higher Vocational Colleges of Philosophy and Social Science Research Project in Jiangsu Universities, China (grant no. 2020SJA0942)" and "Research on Construction of StudentOriented Effective Classroom in Higher Vocational Education of Jiangsu Vocational Education Research Project with Approval of Jiangsu Vocational and Technical Education Association, China (grant no. XHYBLX2021065)".

\section{References}

[1] A. Abbas, M. Choi, J. O. Seo, S. H. Cha, and H. Li, "Effectiveness of immersive virtual reality-based communication for construction projects," KSCE journal of civil engineering, vol. 23, no. 12, pp. 4972-4983, 2019.

[2] C. Ackermann, M. Beggiato, S. Schubert, and J. F. Krems, “An experimental study to investigate design and assessment criteria: what is important for communication between pedestrians and automated vehicles?," applied ergonomics, vol. 75, pp. 272-282, 2019.

[3] S. A. Baker and M. J. Walsh, "'Good Morning Fitfam': top posts, hashtags and gender display on Instagram," New media \& society, vol. 20, no. 12, pp. 4553-4570, 2018.

[4] M. Dhieb, M. Al-Amri, and A. Jamil, "The Digital Urban Atlas of Jeddah: some raised issues and semiological principles," Current urban studies, vol. 7, no. 2, pp. 265-287, 2019.

[5] C. Forceville and S. Paling, "The metaphorical representation of depression in short, wordless animation films," Visual communication, vol. 20, no. 1, pp. 100-120, 2021.

[6] R. Hamaguchi, S. Nematollahi, and D. J. Minter, "Picture of a pandemic: visual aids in the COVID-19 crisis," Journal of Public Health, vol. 42, no. 3, pp. 483-485, 2020.

[7] Z. Jafri, N. Ahmad, M. Sawai, N. Sultan, and A. Bhardwaj, "Digital smile design-an innovative tool in aesthetic dentistry," Journal of oral biology and craniofacial research, vol. 10, no. 2, pp. 194-198, 2020. 
[8] S. Jahnavi and C. Nandini, "Novel multifold secured system by combining multimodal mask steganography and naive based random visual cryptography system for digital communication," Journal of computational and theoretical nanoscience, vol. 17, no. 12, pp. 5279-5295, 2020.

[9] C. Kearns, "Is drawing a valuable skill in surgical practice? 100 surgeons weigh in," Journal of visual communication in medicine, vol. 42, no. 1, pp. 4-14, 2019.

[10] F. Kurasawa, "On humanitarian virality: Kony 2012, or, the rise and fall of a pictorial artifact in the digital age," Visual communication, vol. 18, no. 3, pp. 399-423, 2019.

[11] M.-J. Lee and J.-H. Pae, "Photo-fake conditions of digital landscape representation," Visual communication, vol. 17, no. 1, pp. 3-23, 2018.

[12] A. Lehmuskallio, J. Häkkinen, and J. Seppänen, "Photorealistic computer-generated images are difficult to distinguish from digital photographs: a case study with professional photographers and photo-editors," Visual communication, vol. 18, no. 4, pp. 427-451, 2019.

[13] S. McDonough and E. Colucci, "People of immigrant and refugee background sharing experiences of mental health recovery: reflections and recommendations on using digital storytelling," Visual communication, vol. 20, no. 1, pp. 134156, 2021.

[14] J. Morris, "Exploring the affordances of digital storytelling in a media-arts restorative justice program," Visual communication, vol. 18, no. 2, pp. 205-230, 2019.

[15] J. Ranker, "The sliding of the signified: multimodal sign operations in a youth-created experimental digital video," Visual communication, vol. 17, no. 3, pp. 337-362, 2018.

[16] L. J. Ravelli and T. V. Leeuwen, "Modality in the digital age," Visual communication, vol. 17, no. 3, pp. 277-297, 2018.

[17] K. B. Schloss, Z. Leggon, and L. Lessard, "Semantic discriminability for visual communication," Ieee transactions on visualization and computer graphics, vol. 27, no. 2, pp. 1022-1031, 2021.

[18] J. Sinanan, "Visualising intimacies: the circulation of digital images in the Trinidadian context," Emotion space and society, vol. 31, pp. 93-101, 2019.

[19] A. Srivastava, "Digital art: a revolutionary form of art \& visual communication," International journal of research, vol. 7, no. 11, pp. 83-88, 2019.

[20] A. Taraszkiewicz, "Freehand drawing versus digital design tools in architectural teaching," Australasian journal of engineering education, vol. 23, pp. 100-105, 2021.

[21] C. Valentini, S. Romenti, G. Murtarelli, and M. Pizzetti, "Digital visual engagement: influencing purchase intentions on Instagram," Journal of communication management, vol. 22, no. 4, pp. 362-381, 2018. 